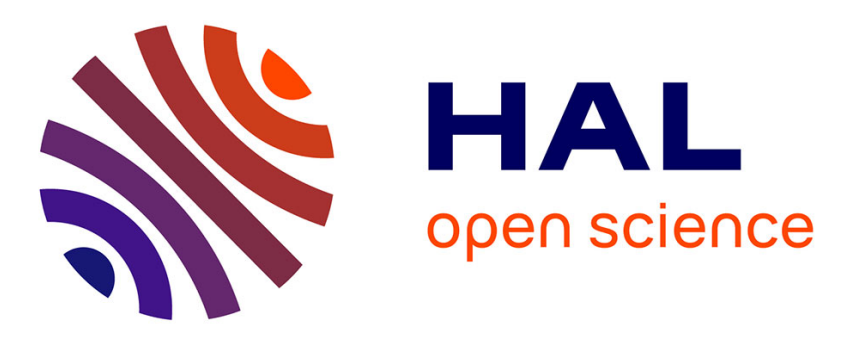

\title{
Potential room-temperature multiferroicity in cupric oxide under high pressure
}

\author{
William Lafargue-Dit-Hauret, Daniel Braithwaite, Andrew D. Huxley, \\ Tsuyoshi Kimura, Andrés Saúl, Xavier Rocquefelte
}

\section{- To cite this version:}

William Lafargue-Dit-Hauret, Daniel Braithwaite, Andrew D. Huxley, Tsuyoshi Kimura, Andrés Saúl, et al.. Potential room-temperature multiferroicity in cupric oxide under high pressure. Physical Review B, 2021, 103 (21), pp.214432. 10.1103/PhysRevB.103.214432 . hal-03281460

\author{
HAL Id: hal-03281460 \\ https://hal.science/hal-03281460
}

Submitted on 29 Oct 2021

HAL is a multi-disciplinary open access archive for the deposit and dissemination of scientific research documents, whether they are published or not. The documents may come from teaching and research institutions in France or abroad, or from public or private research centers.
L'archive ouverte pluridisciplinaire HAL, est destinée au dépôt et à la diffusion de documents scientifiques de niveau recherche, publiés ou non, émanant des établissements d'enseignement et de recherche français ou étrangers, des laboratoires publics ou privés. 


\title{
Potential room-temperature multiferroicity in cupric oxide under high pressure
}

\author{
William Lafargue-Dit-Hauret,, , 2* Daniel Braithwaite, ${ }^{3}$ Andrew D. \\ Huxley, ${ }^{4}$ Tsuyoshi Kimura, ${ }^{5}$ Andres Saúl, ${ }^{6}$ and Xavier Rocquefelte ${ }^{1, \dagger}$ \\ ${ }^{1}$ Univ Rennes, CNRS, ISCR (Institut des Sciences \\ Chimiques de Rennes) UMR 6226, F-35000 Rennes, France \\ ${ }^{2}$ CNRS/Univ. Pau \& Pays Adour / E2S UPPA, \\ Institut des Sciences Analytiques et de Physicochimie pour \\ l'Environnement et les Matériaux - UMR 5254, 64000 Pau, France \\ ${ }^{3}$ Univ. Grenoble Alpes, Grenoble INP, CEA, \\ IRIG-Pheliqs, F-38000 Grenoble, France. \\ ${ }^{4}$ Centre for Science at Extreme Conditions and SUPA, \\ School of Physics and Astronomy, University of Edinburgh, Edinburgh, EH9 3JZ, UK \\ ${ }^{5}$ Department of Advanced Materials Science, \\ University of Tokyo, Kashiwa, Chiba 277-8561, Japan \\ ${ }^{6}$ Aix Marseille Univ, CNRS, CINaM UMR 7325, \\ Campus de Luminy Case 913, 13288 Marseille, France
} (Dated: May 26, 2021) 


\begin{abstract}
$\mathrm{CuO}$, known to be multiferroic $(\mathrm{MF})$ from $T_{L}=213 \mathrm{~K}$ to $T_{N}=230 \mathrm{~K}$ at ambient pressure, has been the subject of debates about its ability to exhibit multiferroicity at room temperature (RT) under high hydrostatic pressure. Here, we address this question based on theoretical and experimental investigations. The influence of hydrostatic pressure on $T_{L}$ and $T_{N}$ has been estimated from ab initio calculations combined with classical Monte-Carlo simulations and a quasi-1D antiferromagnetic analytical model. From the experimental side, electric permittivity anomalies related to ferroelectric transitions have been followed with dielectric measurements on single crystals up to $6.1 \mathrm{GPa}$. We show that the temperature $T_{N}$ below which the MF state forms increases with pressure linearly to higher pressure that hitherto supposed, and indeed based on our calculations should exceed RT above about $20 \mathrm{GPa}$.
\end{abstract}

\title{
I. INTRODUCTION
}

Multiferroic (MF) materials are usually associated with compounds which simultaneously exhibit magnetism and ferroelectricity. The coexistence of both ferroic orders has attracted a considerable attention from industry and academic researchers for several decades [1-4]. Indeed, the development of magnetoelectric materials operating at room temperatures (RT) constitutes the Holy Grail in this field. They could play key roles in magnetic field sensors or memory devices, and also advance in the fundamental understanding of the coupling between the two degrees of freedom. In such compounds, the electric polarization can be controlled through the application of a magnetic field, and an electric field can be used to orientate the macroscopic magnetization. Beyond the difficulty of finding systems in which both properties are coupled at sufficiently high temperatures, some industrial constraints must also be fulfilled: miniaturization, high power efficiency, biocompatibility, low cost, etc.

Thirteen years ago, experimental investigations demonstrated that cupric oxide $\mathrm{CuO}$ exhibits magnetoelectric properties [5]. The ferroelectricity appears to emerge from the incommensurate non-collinear magnetic order (hereafter labelled $A F_{2}$ ) existing between the lock-in temperature $T_{L}$ and the Néel temperature $T_{N}$ located at $213 \mathrm{~K}$ and $230 \mathrm{~K}$, respectively. This incommensurate magnetic phase is characterized by a propagation vector $\boldsymbol{q}=$

* william.lafargue-dit-hauret@univ-pau.fr

$\dagger$ xavier.rocquefelte@univ-rennes1.fr 
$(0.506,0,-0.483)$ [6]. Below $T_{L}, \mathrm{CuO}$ shows a commensurate collinear antiferromagnetic (AFM) order (hereafter labelled $A F_{1}$ ) with all magnetic moments oriented along the crystallographic $\boldsymbol{b}$ axis. The anomalously high Néel temperature for a cuprate system and the significant electric polarization $\left(\sim 100 \mu \mathrm{C} \mathrm{m}^{-2}\right.$ [7]) make this compound a promising candidate for high temperature MF applications. However, it is limited by the small temperature range over which MF is found (i.e., only $17 \mathrm{~K}$ ) that is still far from RT. Experimental studies have demonstrated that the application of a hydrostatic pressure below 2 GPa enlarges the MF stability domain by decreasing $T_{L}$ and increasing $T_{N}$ [8, 9]. Based on the combination of Density Functional Theory (DFT) and effective models (analytical and Monte-Carlo simulations), multiferroicity at RT has been predicted to appear under an applied pressure of about 20-40 GPa [10]. In 2016, Jana et al. reported an experimental investigation which might suggest that $\mathrm{CuO}$ exhibits a $\mathrm{RT}$ ferroelectric polarization at a pressure of only 4.4 GPa [11]. In this study, polycrystalline samples were used without pressure transmitting medium leading to unavoidable non-hydrostatic pressure effects and inhomogeneities during the compression of the sample. In contrast, based on neutron and XRD analyzes performed

on single crystals, Kozlenko et al. claimed that the upper limit of $T_{N}$ would be $260 \mathrm{~K}$ at 38 GPa [12].

The aim of this paper is to discuss the possibility of RT multiferroicity in $\mathrm{CuO}$ through a combined theoretical and experimental investigation. Briefly, we calculated effective magnetic interactions and estimated the transition temperatures using powder experimental atomic structures obtained under hydrostatic pressures ranging from 0 to 38 GPa [12]. Experimentally, we present electric permittivity measurements as a function of temperature and pressure up to 6.1 GPa. The experiments show a continuous widening of the MF stability domain which coupled with the theoretical calculations allow to extrapolate the existence of a MF phase at RT when the pressure approaches $20 \mathrm{GPa}$.

\section{METHODS}

Crystal structure $\mathrm{CuO}$ crystallizes within a $C 2 / c$ monoclinic phase defined by $a=4.695$ $\AA, b=3.436 \AA, c=5.147 \AA$, and $\beta=99.46^{\circ}$ at zero pressure [12]. Cu atoms are located at the center of oxygen square planar environments. These entities, also called plaquettes, originate from an octahedral environment which undergoes a Jahn-Teller distortion. The 
three-dimensional atomic structure of $\mathrm{CuO}$ can be viewed as based on edge-sharing ribbons which are linked together through corners of $\mathrm{CuO}_{4}$. It should be noticed that the related lattice parameters vary non-linearly and anisotropically with pressure and temperature [1316 .

\section{A. Computational details}

First-principles calculations DFT simulations have been carried out using the full-potential LAPW method as implemented within the Wien2k package [17]. The exchange-correlation term was set using the PBE0 on-site hybrid functional [18], which allows to treat a given set of strongly correlated electrons, namely $3 d-\mathrm{Cu}$ orbitals. The Muffin-Tin radii were set to 1.94 and $1.67 a_{0}$ for copper and oxygen atoms, respectively. The basis function expansion was set by the parameter $R K_{\max }=7$. Calculations have been performed on the experimental structures provided in Ref 12. We investigated the electronic properties of the 8 formula unit (f.u.) magnetic cell [19] defined by: $\boldsymbol{a}_{\boldsymbol{m}}=\boldsymbol{a}-\boldsymbol{c}, \boldsymbol{b}_{\boldsymbol{m}}=\boldsymbol{b}$ and $\boldsymbol{c}_{\boldsymbol{m}}=\boldsymbol{a}+\boldsymbol{c}$ where $\boldsymbol{a}, \boldsymbol{b}$ and $\boldsymbol{c}$ are the lattice parameters of the $C 2 / c$ unit cell. Integrations in the first Brillouin zone were performed with a $5 \times 11 \times 6$ Monkhorst-Pack $k$-mesh. For magnetic interactions, we estimated the $J_{i j}$ magnetic exchange couplings by fitting 13 non-equivalent magnetic configurations with an Ising model [20 25]. The following convention has been considered for $J_{i j}$ couplings: a positive sign corresponds to an antiferromagnetic (AFM) character, while a negative sign a ferromagnetic (FM) interaction. We calculated the Magnetocrystalline Anisotropy (MCA) by including the Spin-Orbit Coupling (SOC) as a second-order correction to the total energy.

Bloch spin states The commensurability of the magnetic ground state, dictated by magnetic interactions, has been investigated by studying the dispersion of spin states [26]. The examination of the first ordered state depending on the wave vector $\boldsymbol{k}$ and isotropic exchange couplings $J_{i j}$ was done by solving the eigenvalue problem:

$$
\xi\left(\boldsymbol{k}, J_{i j}\right) \sigma\left(\boldsymbol{k}, J_{i j}\right)=\lambda\left(\boldsymbol{k}, J_{i j}\right) \sigma\left(\boldsymbol{k}, J_{i j}\right)
$$

where $\sigma$ is the spin configuration, $\lambda$ is the energy of the branch, and $\xi$ is the Fourier transform of the exchange integral matrix expressed as:

$$
\xi\left(\boldsymbol{k}, J_{i j}\right)=\sum_{m} J_{i j}\left(\boldsymbol{R}_{m}\right) e^{-2 \pi i \boldsymbol{k} \boldsymbol{R}_{m}}
$$


where $\boldsymbol{R}_{m}$ is the lattice vector separating spins $i$ and $j$.

CMC simulations The pressure vs. temperature MF phase diagram was theoretically investigated by Classical Monte-Carlo (CMC) simulations parametrized by an effective magnetic model expressed as:

$$
H_{\text {model }}=H_{H}+H_{U A}+H_{D M}+H_{M A}
$$

where $H_{H}$ is the Heisenberg term, $H_{U A}$ is an uniaxial anisotropy parameter, $H_{D M}$ is the Dzyaloshinskii-Moriya coupling and $H_{M A}$ is a multiaxial term. Details on the physical meaning and the construction of each term can be found in Ref 10. For each pressure, the magnetic interactions $J_{i j}$ and MCA determined at $0 \mathrm{~K}$ within DFT were injected into the magnetic model. CMC calculations were done on a supercell made of $12^{3}$ spins. Both thermalization and equilibration processes were performed on $10^{6} \mathrm{CMC}$ steps. MF phase transitions were extracted by following the spin current $\boldsymbol{P} \propto\left\langle\boldsymbol{e}_{\boldsymbol{i j}} \times\left(\boldsymbol{S}_{\boldsymbol{i}} \times \boldsymbol{S}_{\boldsymbol{j}}\right)\right\rangle$, which relates the electronic contribution of the electric polarization $\boldsymbol{P}$ to the perpendicularly oriented magnetic moments $\boldsymbol{S}_{\boldsymbol{i}}$ and $\boldsymbol{S}_{\boldsymbol{j}}$ connected by the unit vector $\boldsymbol{e}_{\boldsymbol{i} \boldsymbol{j}}$ [27].

Quasi-1D magnetic model Several experimental studies have concluded that $\mathrm{CuO}$ can be described as a quasi-1D antiferromagnet [28 31] with the Néel temperature $T_{N}$ based on the random phase approximation model of a quasi-1D AFM Heisenberg cubic lattice [32]:

$$
J^{\prime}=\frac{T_{N}}{4 c \sqrt{\ln \left(\alpha J / T_{N}\right)+0.5 \ln \left(\ln \left(\alpha J / T_{N}\right)\right)}}
$$

where $\alpha=2.6$ and $c=0.233$ are numerical parameters, $J^{\prime}$ is an effective inter-chain coupling and $J$ is the intra-chain coupling.

In 2013 [10], we have already used this model to describe the evolution of $T_{N}$ with pressure. We showed that two solutions lead to similar results: (1) adjusting $c$ to 0.284 and keeping $\alpha$ equals to 2.6, (2) adjusting $\alpha$ to 8.4 and keeping $c$ equals to 0.233. In 2017, Kozlenko et al. [12], reconsider our proposition adjusting both $c$ and $\alpha$ values to $c=0.274$ and $\alpha=1.5$. In addition, they considered empirical $J_{i j}$ values, based on the knowledge of superexchange angles.

As in our previous investigation [10], our calculations are based on the $J_{i j}$ values obtained from DFT, taking into account their pressure dependence. We define $J=J_{z}$ and $J^{\prime}=$ $\left(J_{2 a}-J_{x}\right) / 2$ to coincide with the expression of the collinear magnetic ground state energy 
defined as $E_{G S}=J_{z}-J_{x}+J_{2 a}$. To adjust $c$ and $\alpha$ values, we have considered not only the $T_{N}$ value at $0 \mathrm{GPa}$ but also at $5 \mathrm{GPa}$, leading to $c=0.237$ and $\alpha=3.474$.

\section{B. Experimental details}

Dielectric measurements were carried out in a diamond anvil cell (DAC). The technique is described in detail elsewhere [33]. A single crystal of $\mathrm{CuO}$ was cleaved perpendicular to the $\boldsymbol{b}$ axis and polished to a thickness of about $40 \mu \mathrm{m}$. Pieces of size approximately 200x200 $\mu \mathrm{m}$ suitable for the DAC were then cleaved from this piece. A $10 \mu \mathrm{m}$ gold wire was attached to each side of the sample with silver epoxy, which was spread to almost cover the whole surface. The sample wires were connected to 2 co-ax wires. The cell was loaded with liquid argon as the hydrostatic pressure medium, and the pressure was measured in-situ using the ruby fluorescence technique. The capacitance of the sample, proportional to its dielectric constant, was measured with a capacitance bridge at $1 \mathrm{kHz}$ (Andeen-Hagerling AH 2550A). The DAC was placed in a Gifford-McMahon cryocooler where the temperature could be swept from $300 \mathrm{~K}$ to about $20 \mathrm{~K}$.

\section{RESULTS AND DISCUSSIONS}

$J_{i j}$ couplings In the last few years, many investigations have reported theoretical and experimental evaluations of the effective exchange interactions $J_{i j}$ (see Ref 34 for a summary). It should be noted that estimates for $J_{i j}$ vary strongly depending on the effective Hamiltonian used for the experimental fit or the theoretical mapping.

The first step of all our calculations was to estimate the $J_{i j}$ exchange couplings and the Magnetocrytalline Anisotropy (MCA). For this purpose, we used a similar procedure than the one considered in Ref 10. The difference is that instead of using theoretical atomic structures relaxed under a hydrostatic pressure varying from 0 to $200 \mathrm{GPa}$ [10], we used the experimental structures extracted from Ref 12 for pressure values going from 0 to 38 GPa. Here, five magnetic exchange interactions have been considered, depicted in Figure 1. As

shown in Figure 2, their pressure dependence is very similar to the evolution under pressure predicted for the theoretically calculated structures [10].

In short, five $J_{i j}$ exchange interactions (where $i \neq j$ ) are sufficient to describe the magnetic 


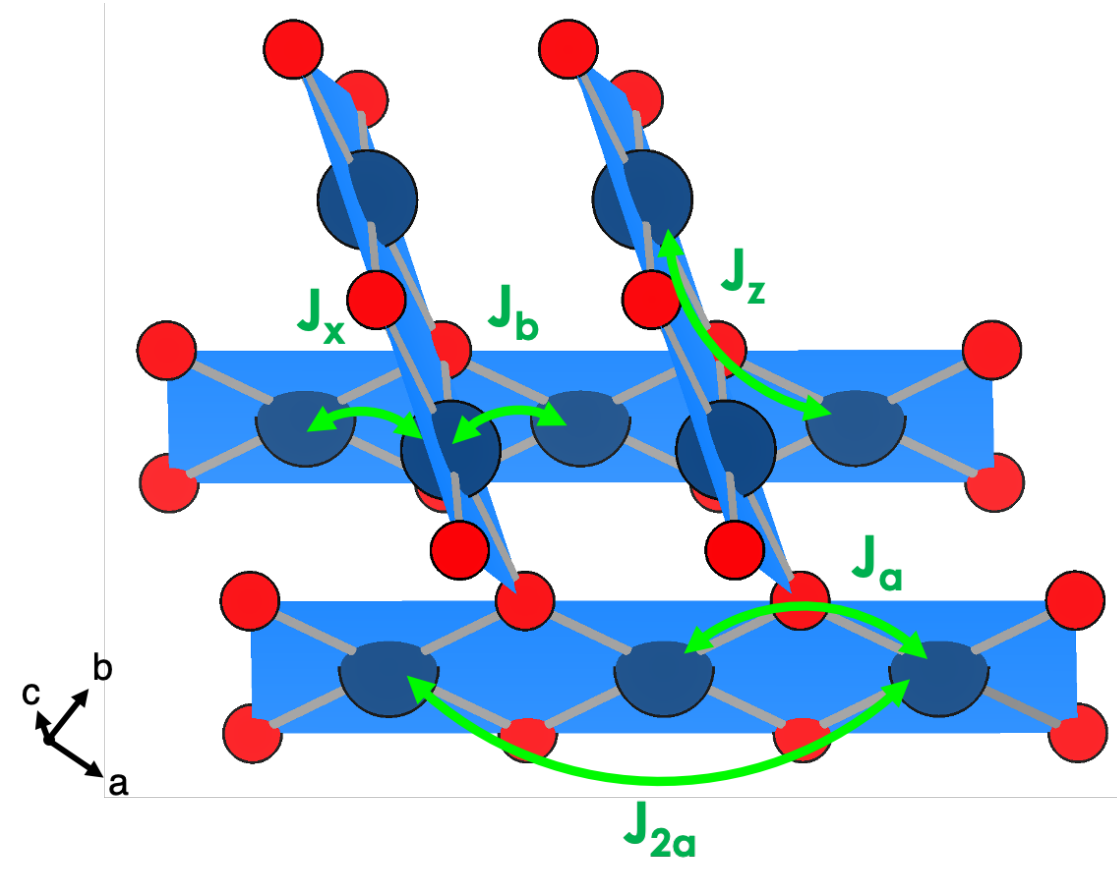

Figure 1. Magnetic exchange couplings considered in this study. The different interactions are highlighted by green arrows. Blue and red spheres indicate copper and oxygen atoms, respectively. Square planar environments are represented in blue.

properties of $\mathrm{CuO}$ [10, 35, 36]. Among them, two are significantly larger, i.e., corner sharing $J_{z}$ and next nearest neighbor intra-ribbon $J_{2 a}$ with values of about 80 and $20 \mathrm{meV}$ at $0 \mathrm{GPa}$, respectively. The three others $\left(J_{x}, J_{a}\right.$ and $\left.J_{b}\right)$ are smaller. Under pressure, $J_{z}$ rises from 78.1 $\mathrm{meV}$ (0 GPa) to $124.6 \mathrm{meV}$ (38 GPa). The second most important interaction corresponds to $J_{2 a}$, which remains roughly unchanged with the pressure varying slightly from 19.7 (0 GPa) to $22.7 \mathrm{meV}$ (38 GPa). Regarding the nearest neighbor intra-ribbon coupling $J_{a}$, the increase of the hydrostatic pressure changes its character from AFM to FM above $~ 3$ GPa. The value of this coupling ranges from $2.6 \mathrm{meV}(0 \mathrm{GPa})$ to $-10.6 \mathrm{meV}$ (38 GPa). The other two inter-ribbon interactions $J_{x}$ and $J_{b}$ become more and more FM with pressure, reaching -13.6 and $-6.8 \mathrm{meV}$ at $38 \mathrm{GPa}$, respectively.

To our knowledge, the experimental pressure dependence of the $J_{i j}$ exchange interactions has not been reported, so far. We will thus compare our calculations with the ambient pressure neutron scattering experimental data [34], as summarized in Table I. The authors applied constraints to simplify their Hamiltonian, leading to differences with our Hamiltonian. In our case, we consider not only the $\mathrm{Cu}-\mathrm{Cu}$ interatomic distances to define a magnetic 


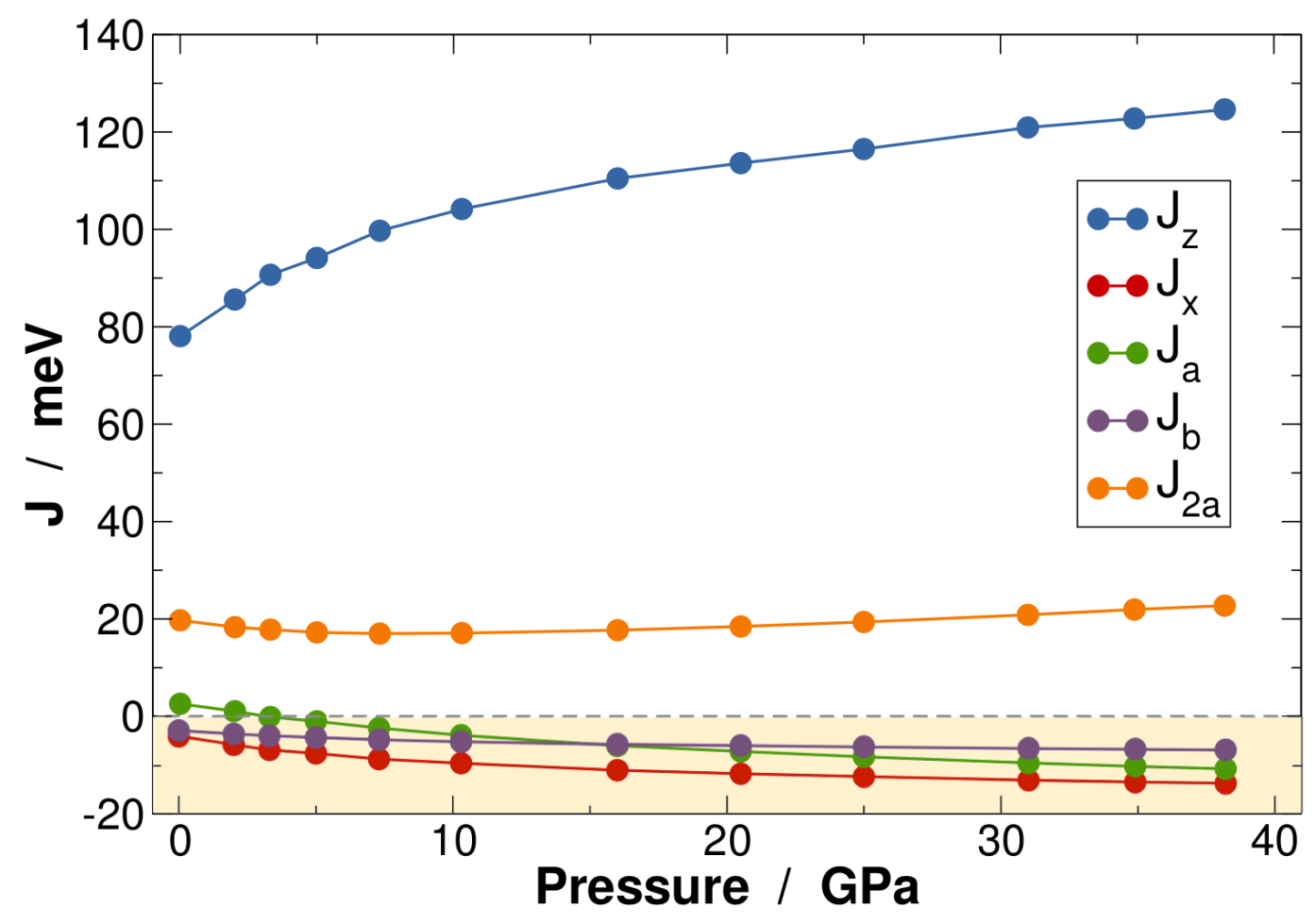

Figure 2. Magnetic exchange interactions vs. hydrostatic pressure. Positive and negative signs correspond to antiferromagnetic and ferromagnetic interactions, respectively.

exchange path, but also the overlap of the magnetic orbitals (bond or dihedral angles). As a consequence, a correction of a factor of 2 must be applied to $J_{2 a}$ and $J_{a}$ deduced from neutron scattering to compare with our results, leading to 6.34 and $\pm 5.0 \mathrm{meV}$, respectively. The agreement with DFT is good except for $J_{2 a}$ for which DFT gives a higher value (19.7 $\mathrm{meV}) . J_{2 a}$ corresponds to a super-superexchange interaction between two coplanar $\mathrm{CuO}_{4}$ plaquettes. As a consequence, the overlap between the magnetic orbitals, through the $2 p$-O states, is optimal, and thus a large value is not counter-intuitive. Interpreting this deviation is not straightforward. From the point of view of the neutron scattering experiments, no clear evidence of a coupling of about $20 \mathrm{meV}$ has been identified. Only one path in the reported magnetic dispersion probes the effect of $J_{2 a}$, leading the authors to conclude that $J_{2 a}$ is necessary to reproduce the minimum in the dispersion at the $X$ point, corresponding to $\boldsymbol{q}=(1,1 / 2,0)$. However, the effect of $J_{2 a}$ is strongest in the direction from $(1 / 2,0,-1 / 2)$ to $(1,1 / 2,-1 / 2)$, and the last point is not given in the reported data. 


\begin{tabular}{l|ccccc} 
& $J_{z}$ & $J_{x}$ & $J_{a}$ & $J_{b}$ & $J_{2 a}$ \\
\hline$d_{C u-C u}^{0 G P a}$ & 3.757 & 3.186 & 2.909 & 3.095 & 5.819 \\
This work & 78.1 & -4.0 & 2.6 & -2.9 & 19.7 \\
Exp. [34] & $91.4(5)$ & $-3.73(3)$ & $\pm 2.50(18)$ & $\pm 3.10(18)$ & $3.17(3)$
\end{tabular}

Table I. Magnetic exchange interactions (in meV) computed for the 0 GPa structure. The interatomic distances are given in $\AA$, and the inelastic neutron values from Ref 34 are also reported.

$M C A$ We estimated the MCA by including the SOC. We found that the intensity of MCA is weakly affected even under relatively high pressures (see Supplemental Materials [37]). Also, we shown that the easy magnetization axis is unchanged under pressure up to $38 \mathrm{GPa}$, the magnetic moments being still preferentially oriented along the [010] direction, in the lines of experimental observations at $0 \mathrm{GPa}$ [6]. These results closely agree with our previous predictions [10]. One may notice that the intermediate magnetization axis, computed at ambient pressure, is found within the $a c$ plane with an angle of $60^{\circ}$ with respect to the $\boldsymbol{c}$ crystallographic axis. This prediction significantly differs from the experimental value of $28.8(8)^{\circ}$ revealed by neutron measurements [38]. The understanding of this difference is the subject of ongoing collaborative works combining Torque magnetometry analysis and DFT calculations.

Incommensurate magnetic structure Neutron scattering has shown that the low-temperature ground state magnetic structure $A F_{1}$, with a propagation vector $\boldsymbol{q}=(0.5,0,-0.5)$, is commensurate with the crystallographic cell, while the $A F_{2}$ magnetic structure is incommensurate at ambient pressure with $\boldsymbol{q}=(0.526,0,-0.483)$ [6]. Based on the Freiser method [26], the knowledge of the magnetic exchange couplings allows us to estimate the total spin exchange energy for a given propagation vector $\boldsymbol{q}$. Figure 3 depicts the Bloch spin states of minimal energy within the $\left(\boldsymbol{q}_{\boldsymbol{x}}, \boldsymbol{q}_{\boldsymbol{z}}\right)$ plane for $0 \mathrm{GPa}$. As can be seen, the maxima are found close to the middle of the edges of the Birillouin zone $( \pm 0.5,0)$ and $(0, \pm 0.5)$ and the minima close to the corners $( \pm 0.5, \pm 0.5)$. Such a situation implies the magnetic cell can only be described by a crystal supercell, which follows nicely the experimental observations [6].

We obtained $\boldsymbol{q}=(0.53,0,-0.45)$ at $0 \mathrm{GPa}$, in good agreement with the $A F_{2}$ experimental 
(a)

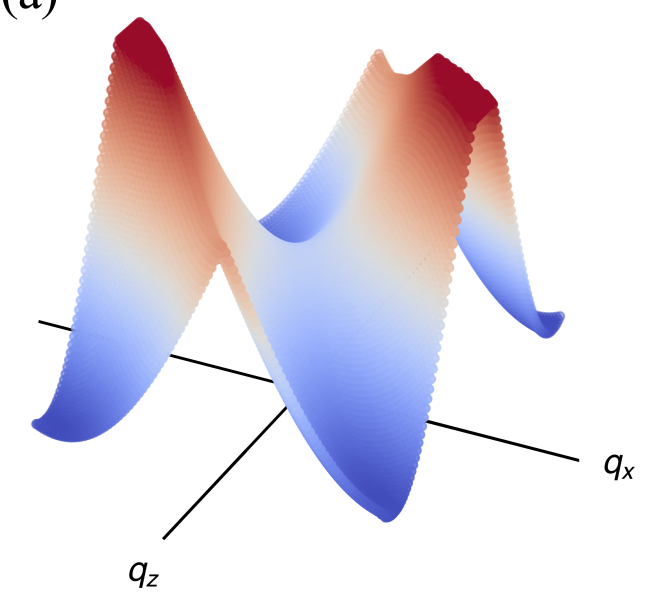

(b)

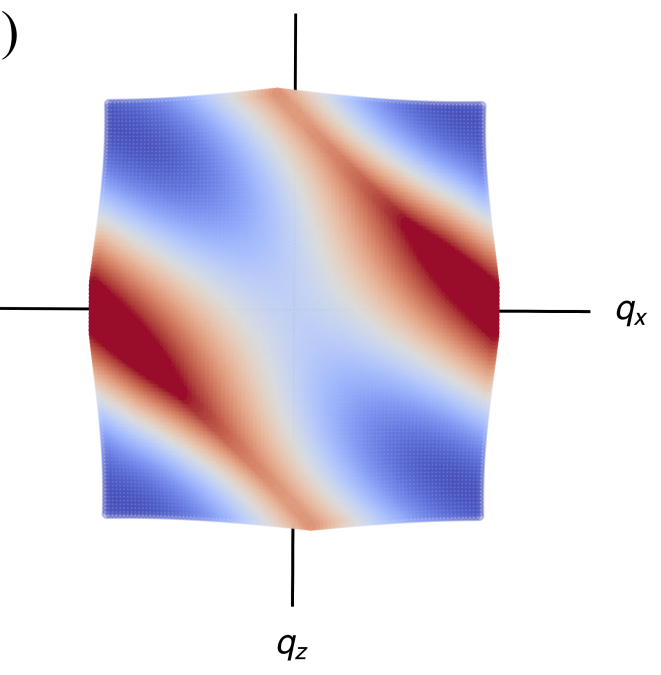

Figure 3. (a) Diagonal and (b) top view of the dispersion of the magnetic ground state within the $\left(\boldsymbol{q}_{\boldsymbol{x}}, \boldsymbol{q}_{\boldsymbol{z}}\right)$ plane at $0 \mathrm{GPa}$. Red color highlights highest energies, while dark blue regions evidence energy minima.

propagation vector [6, 38, 39]. We have then used the Freiser approach to predict the evolution of the magnetic structure under pressure. At $38 \mathrm{GPa}$, we found $\boldsymbol{q}=(0.50,0$, 0.47), suggesting that the magnetic structure of $\mathrm{CuO}$ remains incommensurate up to roughly $40 \mathrm{GPa}$.

Finally, let us note that based on a similar analysis, Dai et al. [40] concluded that the incommensurability of the magnetic structure originates from the $J_{b}$ interaction. By decomposing our Ising hamiltonian and estimating the impact of each contribution, we found that not only $J_{b}$ but also $J_{a}$ take part in this spin spiral behavior.

MF phase diagram The theoretical estimation of the variation of the Néel temperature $T_{N}$ with pressure has been obtained using two different approaches, i.e., by performing CMC simulations and by using an analytical model. Let's first discuss our results deduced from CMC calculations, which were performed using the effective magnetic model expressed in Equation 3 with similar settings to Ref 10. More specifically, the Heisenberg term $H_{H}$ aims to describe the long-range magnetic ordering through exchange interactions. The $H_{U A}$ uniaxial contribution was used to stabilize the $A F_{1}$ phase at low temperature, while the $H_{D M}$ non-collinear term allows incorporation of the Dzyaloshinskii-Moriya coupling to favor 


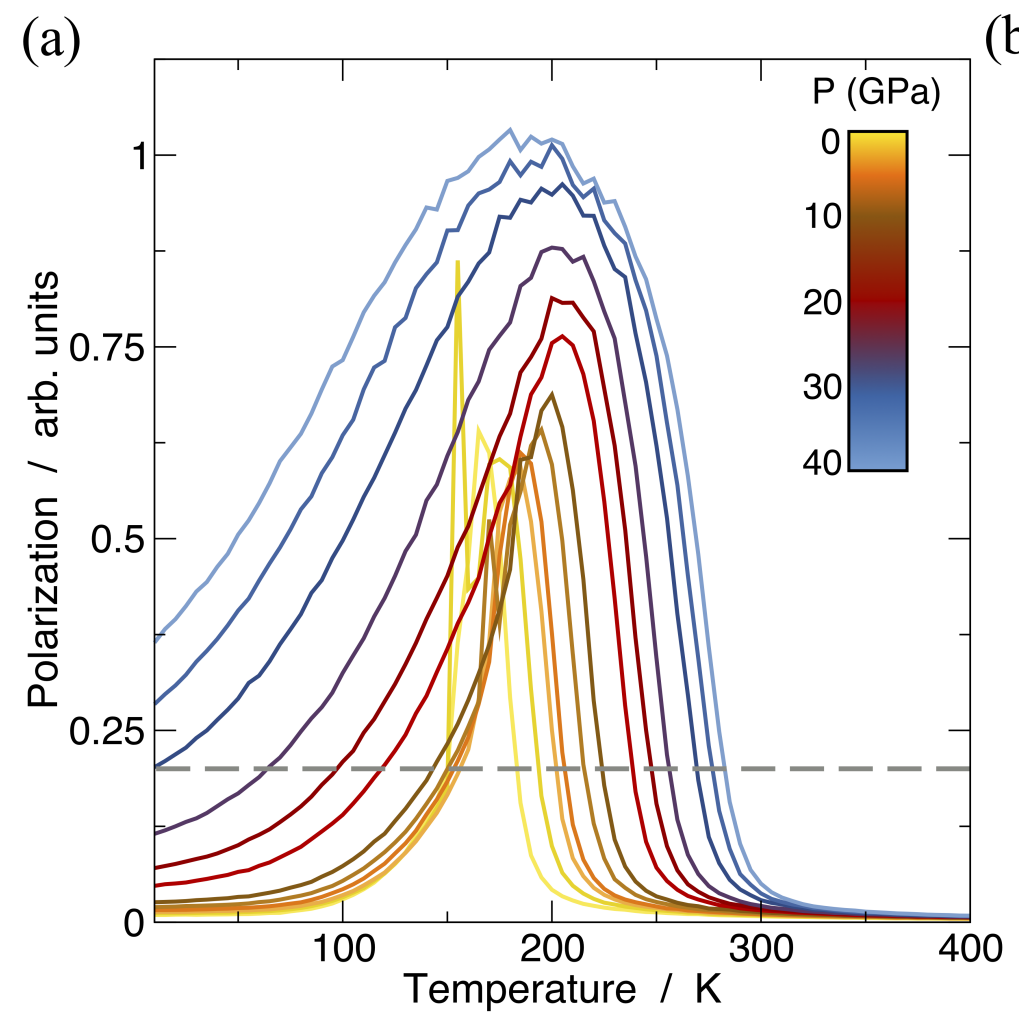

(b)

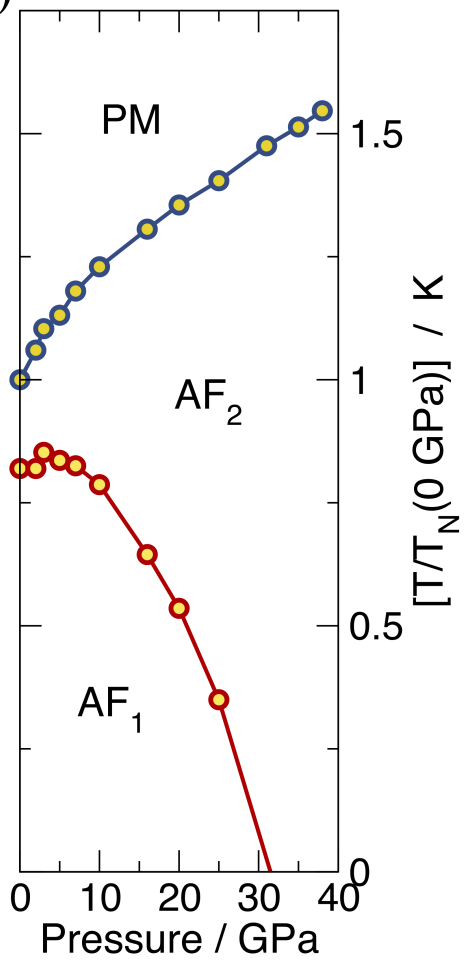

Figure 4. CMC results. (a) Electric polarization vs. temperature for different pressure values. The electric polarization was estimated by $\boldsymbol{P} \propto\left\langle\boldsymbol{e}_{\boldsymbol{i} \boldsymbol{j}} \times\left(\boldsymbol{S}_{\boldsymbol{i}} \times \boldsymbol{S}_{\boldsymbol{j}}\right)\right\rangle$. The gray dashed line is the reference arbitrarily chosen to estimate the MF phase transitions. (b) MF phase diagram. $T_{L}$ and $T_{N}$ variations are represented by red and blue lines, respectively, and normalized at the estimated $\mathrm{CMC} T_{N}$ value at $0 \mathrm{GPa}$.

the $A F_{2}$ phase. Finally, the $H_{M A}$ multiaxial part constrains the magnetic moments within the $(\boldsymbol{b}, \boldsymbol{c})$ plane. By following the evolution of the electric polarization for each pressure step (see Figure 4a), the magnetic phase diagram has been evaluated and is presented in Figure 4b.

Firstly, we found that the electric polarization induced by the non-collinear orientation of magnetic moments increases with pressure. Secondly, the MF stability domain expands, i.e., $T_{L}$ decreases while $T_{N}$ increases, with pressure. These results still agree with our previous predictions, which were based on theoretical structures. Here, we showed that using the experimental refined structures, CMC simulations predict a continuous increase of $T_{N}$ without reaching a plateau as suggested by Kozlenko et al. [12]. 
Experimental dielectric measurements In parallel, we measured the electric permittivity as function of temperature up to 6.1 GPa. In Figure 5, we show the dielectric constant curves for different pressures. A smooth but significant decrease of $\varepsilon$ ' below $100 \mathrm{~K}$ is present at all pressure steps. Such behavior has also been reported by Zheng et al. 41], attributed to a coupling between the magnetic and vibrational degrees of freedom. At RT, $\varepsilon^{\prime}$ is found to increase monotonically with pressure, similar to the initial increase reported by Jana et al. [11. However, we do not retrieve the sharp decrease of the dielectric constant that they observed at $4.4 \mathrm{GPa}$ and assigned to the ferroelectric transition. Also, it is not clear why the transition would induce such a large drop of $\varepsilon^{\prime}$ as this is not the case at ambient pressure. From our data, the $T_{N}$ transition is clearly observed at all pressures as a sharp step, which is reproducible between the cooling and warming cycles. For temperatures ranging between $T_{N}$ and $T_{L}$, the measurement shows hysteretic and non-reproducible behaviour as shown in the inset. $T_{L}$ can however be reliably detected as a small anomaly better seen in the warming curves. To reveal the two transitions, we show in Figure 6 previous curves after subtraction of a polynomial background and with an offset for the different pressures.

From our measurements, the experimental lower and upper limits of spin-spiral stability domain, i.e., $T_{L}$ and $T_{N}$, are represented by red circles in Figure 7. In addition, this figure shows the experimental data of Chatterji et al. (open red diamond) and our theoretical predictions. Two types of calculations are represented: (1) the CMC data which have been shifted to fit the experimental $T_{N}$ value at $0 \mathrm{GPa}$; (2) an analytical model suited for $S=1 / 2$ quasi-1D Heisenberg antiferromagnets. First of all, the CMC data are not expected to provide quantitative results, but general trends. In contrast, the analytical model, if properly defined, should lead to quantitative results. Figure 7 a shows a relatively good agreement between experiments and theory, confirming the increase (decrease) of $T_{N}\left(T_{L}\right)$ with pressure, and thus the stabilization of the spin-spiral magnetic phase, which exhibits multiferroicity. Our simulations suggest that $T_{N}$ may reach $\mathrm{RT}$ at a pressure value of about $20 \mathrm{GPa}$, in agreement with our previous prediction.

A closer look at the data (Figure $7 \mathrm{~b}$ ) evidences some deviations in the $\mathrm{CMC}$ theoretical data compared to the experimental points, in particular for the evolution of $T_{L}$ in the pressure range $[0,2] \mathrm{GPa}$. It is probably due to the approximations made in the CMC model to describe the non-collinearity in $A F_{2}$ with the effective $H_{D M}$ and $H_{M A}$ terms. This 


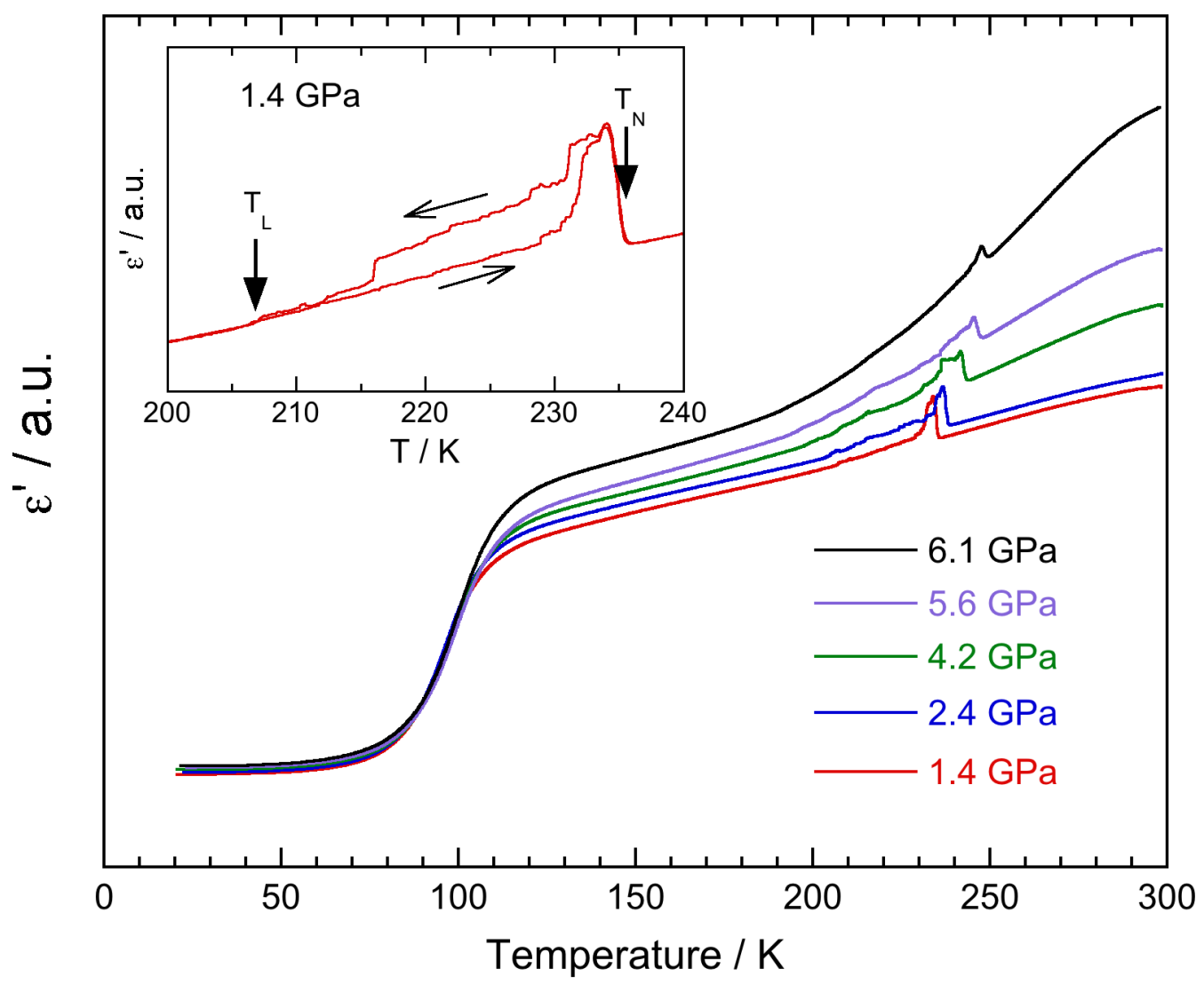

Figure 5. Dielectric constant curves of single crystal $\mathrm{CuO}$ for $\boldsymbol{E} / / \boldsymbol{b}$ from high pressure capacitance measurements with increasing temperature. The inset shows the region of the two magnetic phase transitions for $1.4 \mathrm{GPa}$, indicated by the arrows. The upper transition $\left(T_{N}\right)$ appears as a sharp step reproducible on warming and cooling. The lower transition $\left(T_{L}\right)$ appears as a much smaller anomaly. The region between $T_{L}$ and $T_{N}$ shows marked hysteresis between the cooling and warming cycles, with irreproducible behaviour on cooling.

trend would be improved by estimating such couplings with further non-collinear ab initio calculations, which is beyond the scope of this paper.

As shown in Figure 7, this experimental study is consistent with previous experimental results obtained up to $\sim 2 \mathrm{GPa}$ [8], and confirms our predictions of a continuous widening of the MF phase with pressure. In details, we find quasi-linear variations of $T_{L}$ and $T_{N}$ over the studied pressure range, with $\left(d T_{L}\right) / d P=-3.7 \mathrm{~K} \mathrm{GPa}^{-1}$ and $\left(d T_{N}\right) / d P=3.0 \mathrm{~K} \mathrm{GPa}^{-1}$. This strengthens our theoretical predictions on a significant increase of $T_{N}$ for $\mathrm{CuO}$ under hydrostatic pressure without expected saturation as suggested by Kozlenko et al. [12]. Thus, 


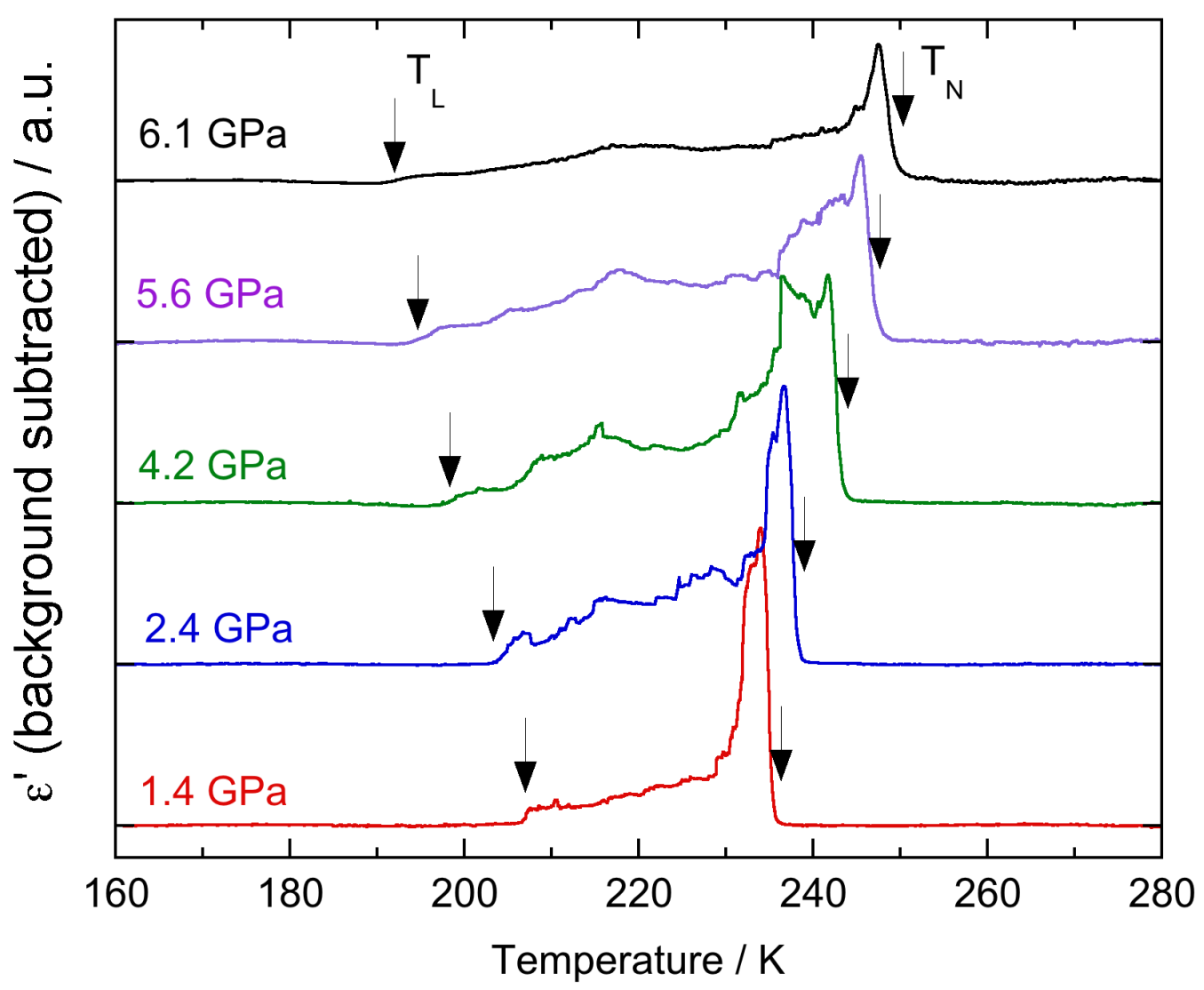

Figure 6. Zoom of the dielectric constant curves shown in Figure 5 after subtraction of an arbitrary polynomial background to reveal $T_{N}$ and $T_{L}$ transitions evidenced by arrows. Curves have been shifted for clarity.

these results support the hypothesis MF properties can be observed at RT.

\section{CONCLUSION}

We combined theoretical and experimental methods to follow the stability domain of the $\mathrm{MF}$ phase of $\mathrm{CuO}$ under hydrostatic pressure. From the theoretical side, we confirm our previous predictions using the refined atomic structures up to about $38 \mathrm{GPa}$ in Ref 12 . More specifically, $T_{L}$ decreases while $T_{N}$ increases under pressure. Experimental dielectric anomalies have been measured up to $6.1 \mathrm{GPa}$. We observed $T_{L}\left(T_{N}\right)$ linearly decreases (increases) with pressure, without detecting any saturation phenomenon. Together these results strongly support the possibility of $\mathrm{RT}$ multiferroicity in $\mathrm{CuO}$ under a pressure of about $20 \mathrm{GPa}$. 

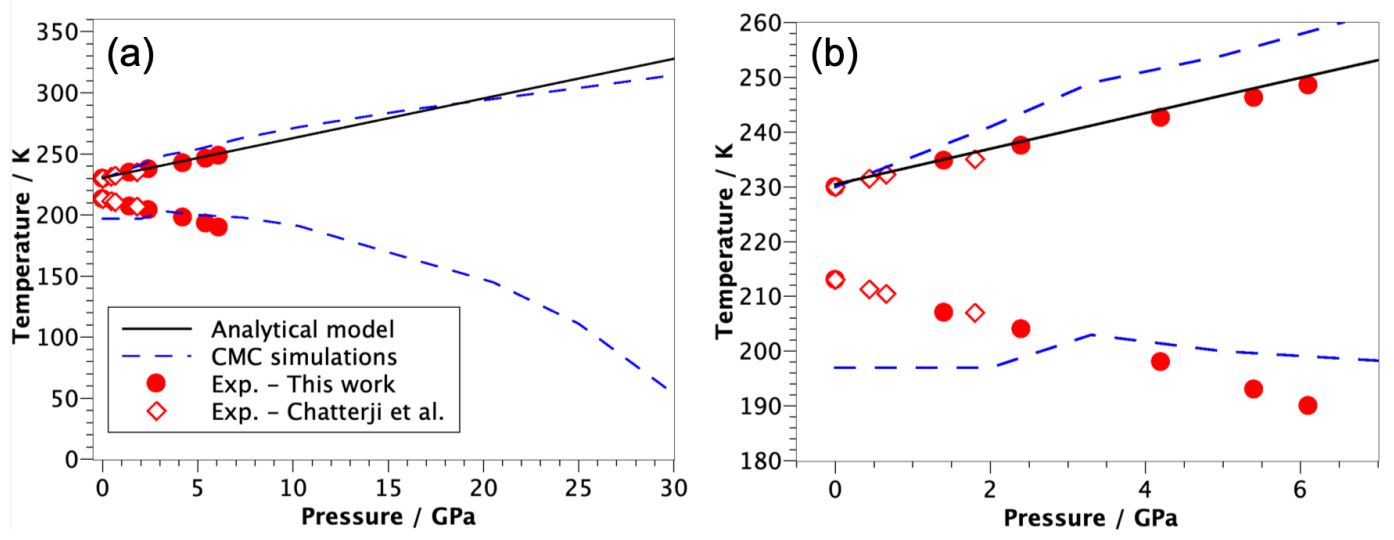

Figure 7. (a) Temperature-Pressure phase diagram of $\mathrm{CuO}$. (a) Experimental values extracted from our dielectric measurements (red plain circles) and from Chatterji et al. [8] (red open circles). Our theoretical estimated values from CMC and the analytical expression (Equation 4) are represented by black line and blue dashed lines, respectively. Our CMC simulations have been adjusted to fit the experimental Néel temperature at $0 \mathrm{GPa}$. (b) Zoom in the pressure range from 0 to $7 \mathrm{GPa}$ to emphasize the experimental/theoretical deviations.

\section{ACKNOWLEDGMENTS}

The theoretical work was granted access to the HPC resources of [TGCC/CINES/IDRIS] under the allocation 2017-A0010907682 made by GENCI. W.L.-D.-H. and X.R. also acknowledge the CCIPL (Centre de Calcul Intensif des Pays de la Loire) for computing facilities, S. Kumar for helpful discussions, and M. Herak for fruitful discussions and comments on the manuscript. D.B., X.R., and A.S. acknowledge funding from the French National Research agency (ANR - Grant ANR-19-CE08-0013-02; HTHPCM Project). T.K. was supported by JSPS KAKENHI under grant numbers JP17H01143 and JP19H05823. D.B. and A.D.H. acknowledge support from EPSRC grant EP/R013004/1.

[1] T. Kimura, Spiral Magnets as Magnetoelectrics, Annual Review of Materials Research 37, 387 (2007)

[2] J. F. Scott, Room-temperature multiferroic magnetoelectrics, NPG Asia Mater 5, e72 (2013). 
[3] S. Dong, J.-M. Liu, S.-W. Cheong, and Z. Ren, Multiferroic materials and magnetoelectric physics: symmetry, entanglement, excitation, and topology, Advances in Physics 64, 519 (2015).

[4] M. Fiebig, T. Lottermoser, D. Meier, and M. Trassin, The evolution of multiferroics, Nature Reviews Materials 1, 16046 (2016).

[5] T. Kimura, Y. Sekio, H. Nakamura, T. Siegrist, and A. P. Ramirez, Cupric oxide as an inducedmultiferroic with high- $\mathrm{T}_{c}$, Nature Materials 7, 291 (2008), number: 4 Publisher: Nature Publishing Group.

[6] J. B. Forsyth, P. J. Brown, and B. M. Wanklyn, Magnetism in cupric oxide, J. Phys. C: Solid State Phys. 21, 2917 (1988).

[7] P. Babkevich, A. Poole, R. D. Johnson, B. Roessli, D. Prabhakaran, and A. T. Boothroyd, Electric field control of chiral magnetic domains in the high-temperature multiferroic $\mathrm{CuO}$, Phys. Rev. B 85, 134428 (2012).

[8] T. Chatterji, P. J. Brown, and J. B. Forsyth, High pressure neutron diffraction investigation of $\mathrm{CuO}$, J. Phys.: Condens. Matter 17, S3057 (2005).

[9] M. Ohashi, A. Tashiro, G. Oomi, E. Maeda, and X.-G. Zheng, Effect of pressure on the magnetic phase transition in cupric oxide, Phys. Rev. B 73, 134421 (2006).

[10] X. Rocquefelte, K. Schwarz, P. Blaha, S. Kumar, and J. van den Brink, Room-temperature spin-spiral multiferroicity in high-pressure cupric oxide, Nat Commun 4, 2511 (2013).

[11] R. Jana, P. Saha, V. Pareek, A. Basu, S. Kapri, S. Bhattacharyya, and G. D. Mukherjee, High Pressure Experimental Studies on CuO: Indication of Re-entrant Multiferroicity at Room Temperature, Scientific Reports 6, 31610 (2016).

[12] D. P. Kozlenko, K. Drużbicki, S. E. Kichanov, E. V. Lukin, H.-P. Liermann, K. V. Glazyrin, and B. N. Savenko, Anomalous lattice compression and magnetic ordering in $\mathrm{CuO}$ at high pressures: A structural study and first-principles calculations, Phys. Rev. B 95, 054115 (2017).

[13] M. Malinowski, S. Åsbrink, and A. Kvick, A high-pressure single-crystal X-ray diffraction study of copper oxide using synchrotron radiation, High Pressure Research 4, 429 (1990).

[14] H. Ehrenberg, J. A. McAllister, W. G. Marshall, and J. P. Attfield, Compressibility of copperoxygen bonds: a high-pressure neutron powder diffraction study of $\mathrm{CuO}$, J. Phys.: Condens. Matter 11, 6501 (1999). 
[15] H. Yamada, X.-G. Zheng, Y. Soejima, and M. Kawaminami, Lattice distortion and magnetolattice coupling in $\mathrm{CuO}$, Phys. Rev. B 69, 104104 (2004).

[16] X. G. Zheng, H. Kubozono, H. Yamada, K. Kato, Y. Ishiwata, and C. N. Xu, Giant negative thermal expansion in magnetic nanocrystals, Nat Nano 3, 724 (2008).

[17] P. Blaha, K. Schwarz, G. K. H. Madsen, D. Kvasnicka, J. Luitz, R. Laskowsk, F. Tran, L. Marks, and L. Marks, WIEN2k: An Augmented Plane Wave Plus Local Orbitals Program for Calculating Crystal Properties (Techn. Universitat, 2019).

[18] F. Tran, P. Blaha, K. Schwarz, and P. Novák, Hybrid exchange-correlation energy functionals for strongly correlated electrons: Applications to transition-metal monoxides, Phys. Rev. B 74, $155108(2006)$.

[19] B. X. Yang, J. M. Tranquada, and G. Shirane, Neutron scattering studies of the magnetic structure of cupric oxide, Phys. Rev. B 38, 174 (1988).

[20] H. Xiang, C. Lee, H.-J. Koo, X. Gong, and M.-H. Whangbo, Magnetic properties and energymapping analysis, Dalton Trans. 42, 823 (2012).

[21] G. Radtke, A. Saúl, H. a. Dabkowska, G. M. Luke, and G. a. Botton, Interplay between Structural, Electronic, and Magnetic Degrees of Freedom in $\mathrm{Sr}_{3} \mathrm{Cr}_{2} \mathrm{O}_{8}$, Phys. Rev. Lett. 105, $036401(2010)$

[22] A. Saúl, D. Vodenicarevic, and G. Radtke, Theoretical study of the magnetic order in $\alpha$ $\mathrm{CoV}_{2} \mathrm{O}_{6}$, Phys. Rev. B 87, 024403 (2013).

[23] A. Saúl and G. Radtke, Density functional approach for the magnetism of $\beta$-TeVO, Phys. Rev. B 89, 104414 (2014).

[24] M. Okada, H. Tanaka, N. Kurita, K. Johmoto, H. Uekusa, A. Miyake, M. Tokunaga, S. Nishimoto, M. Nakamura, M. Jaime, G. Radtke, and A. Saúl, Quasi-two-dimensional Bose-Einstein condensation of spin triplets in the dimerized quantum magnet $\mathrm{Ba}_{2} \mathrm{CuSi}_{2} \mathrm{O}_{6} \mathrm{Cl}_{2}$, Phys. Rev. B 94, 094421 (2016), 1609.00438.

[25] A. Saúl, N. Gauthier, R. M. Askari, M. Côté, T. Maris, C. Reber, A. Lannes, D. Luneau, M. Nicklas, J. M. Law, E. L. Green, J. Wosnitza, A. D. Bianchi, and A. Feiguin, Unconventional field induced phases in a quantum magnet formed by free radical tetramers, Phys. Rev. B 97, $064414(2018)$.

[26] M. J. Freiser, Thermal Variation of the Pitch of Helical Spin Configurations, Phys. Rev. 123, 2003 (1961). 
[27] H. Katsura, N. Nagaosa, and A. V. Balatsky, Spin Current and Magnetoelectric Effect in Noncollinear Magnets, Phys. Rev. Lett. 95, 057205 (2005).

[28] J. W. Loram, K. A. Mirza, C. P. Joyce, and A. J. Osborne, Specific-Heat Evidence for Quasi-1D Magnetic Order in $\mathrm{CuO}$, EPL 8, 263 (1989).

[29] A. T. Boothroyd, A. Mukherjee, S. Fulton, T. G. Perring, R. S. Eccleston, H. A. Mook, and B. M. Wanklyn, High-energy magnetic excitations in $\mathrm{CuO}$, Physica B: Condensed Matter Proceedings of the First European Conference on Neutron Scattering, 234, 731 (1997).

[30] T. Shimizu, T. Matsumoto, A. Goto, K. Yoshimura, and K. Kosuge, Spin susceptibility of the quasi-one-dimensional antiferromagnet $\mathrm{CuO}$, Physica B: Condensed Matter 259-261, 573 (1999).

[31] T. Shimizu, T. Matsumoto, A. Goto, T. V. Chandrasekhar Rao, K. Yoshimura, and K. Kosuge, Spin susceptibility and superexchange interaction in the antiferromagnet $\mathrm{CuO}$, Phys. Rev. B 68, $224433(2003)$.

[32] C. Yasuda, S. Todo, K. Hukushima, F. Alet, M. Keller, M. Troyer, and H. Takayama, Néel Temperature of Quasi-Low-Dimensional Heisenberg Antiferromagnets, Phys. Rev. Lett. 94, $217201(2005)$

[33] J. Mokdad, G. Knebel, C. Marin, J.-P. Brison, I. Matei, and D. Braithwaite, Probing insulators under pressure, Review of Scientific Instruments 91, 093902 (2020).

[34] H. Jacobsen, S. M. Gaw, A. J. Princep, E. Hamilton, S. Tóth, R. A. Ewings, M. Enderle, E. M. H. Wheeler, D. Prabhakaran, and A. T. Boothroyd, Spin dynamics and exchange interactions in $\mathrm{CuO}$ measured by neutron scattering, Physical Review B 97, 144401 (2018), publisher: American Physical Society.

[35] X. Rocquefelte, M.-H. Whangbo, A. Villesuzanne, S. Jobic, F. Tran, K. Schwarz, and P. Blaha, Short-range magnetic order and temperature-dependent properties of cupric oxide, J. Phys.: Condens. Matter 22, 045502 (2010).

[36] X. Rocquefelte, K. Schwarz, and P. Blaha, Theoretical Investigation of the Magnetic Exchange Interactions in Copper(II) Oxides under Chemical and Physical Pressures, Scientific Reports $\mathbf{2}, 10.1038 /$ srep00759 (2012).

[37] See Supplemental Material at [URL] for more details on calculated MCA under hydrostatic pressure up to $38 \mathrm{GPa}$. 
[38] P. J. Brown, T. Chattopadhyay, J. B. Forsyth, and V. Nunez, Antiferromagnetism in CuO studied by neutron polarimetry, J. Phys.: Condens. Matter 3, 4281 (1991).

[39] M. Ain, A. Menelle, B. M. Wanklyn, and E. F. Bertaut, Magnetic structure of CuO by neutron diffraction with polarization analysis, J. Phys.: Condens. Matter 4, 5327 (1992).

[40] D. Dai, H.-J. Koo, and M.-H. Whangbo, Investigation of the Incommensurate and Commensurate Magnetic Superstructures of $\mathrm{LiCuVO}_{4}$ and $\mathrm{CuO}$ on the Basis of the Isotropic Spin Exchange and Classical Spin Approximations, Inorg. Chem. 43, 4026 (2004).

[41] X. G. Zheng, Y. Sakurai, Y. Okayama, T. Q. Yang, L. Y. Zhang, X. Yao, K. Nonaka, and C. N. $\mathrm{Xu}$, Dielectric measurement to probe electron ordering and electron-spin interaction, Journal of Applied Physics 92, 2703 (2002), publisher: American Institute of Physics. 